\title{
PARAVERTEBRAL BLOCK VERSUS ERECTOR SPINAE PLANE BLOCK IN PATIENTS UNDERGOING BREAST CANCER SURGERY: A FEASIBILITY STUDY
}

RJH Smits $M D^{1}$, MGE Fenten $M D^{1}$, GPG Filippini-de Moor MD2, GJ van Geffen MD PhD'

${ }^{1}$ Department of Anesthesiology, pain- and palliative medicine, Radboudumc Nijmegen, the Netherlands

${ }^{2}$ Department of Anesthesiology, Bernhoven hospital Uden, the Netherlands

\section{Introduction}

Surgically treated breast cancer patients have a very high incidence of chronic pain. A risk factor for chronification of pain is severe postoperative pain. Paravertebral (PVB) and erector spinae block (ESPB) are effective regional anesthetic techniques after thoracic surgery. In this study we evaluated these techniques for providing postoperative pain relief after breast surgery.

\section{Material and methods}

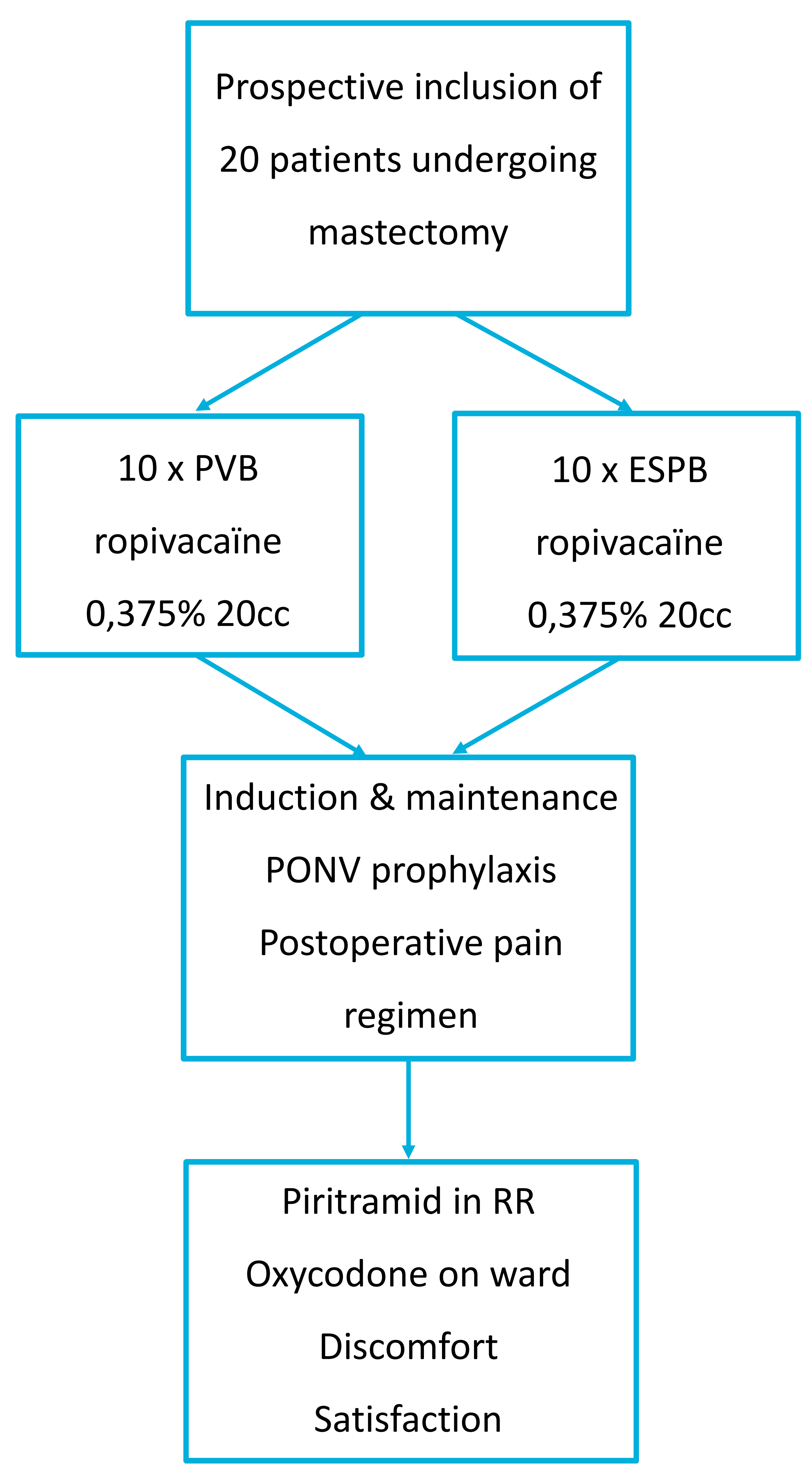

\section{Results}

\begin{tabular}{lll} 
& PVB & ESPB \\
\hline Piritramid in mg & $8(0-20)$ & $4,5(0-20)$ \\
Oxycodone in mg & $5,65(0-20)$ & $1(0-5)$ \\
\hline Discomfort (1-10) & $3(0-6)$ & $1,7(0-4)$ \\
Satisfaction (1-10) & $6,75(1-10)$ & $8,6(7-10)$
\end{tabular}

Table 1. Results of PVB vS ESPB on piritramid and oxycodone consumption in the first 24 hours, discomfort during the intervention, overall satisfaction on pain regimen

\section{Discussion}

Although the study is underpowered for statistical comparisons, higher patient satisfaction and less discomfort during block placement was found in the ESPB group. Together with a better safety profile the results consistently favor the ESPB.

Randomized and adequately powered studies are necessary to confirm these preliminary results.

\section{Conclusion}

- Opioid consumption in ESPB is comparable to PVB

- Patients undergoing ESPB experience little discomfort during the intervention

- Patients undergoing ESPB are very satisfied about the postoperative pain regimen 